\title{
Malignant ureteral obstruction
}

\begin{abstract}
Extrinsic ureteral obstruction secondary to malignancy can be difficult for the urologist to manage. We present a case of ureteral obstruction by locally advanced cervical cancer in a 52-year-old woman. Literature review reflects a lack of randomized clinical data to support a specific treatment strategy, suggesting that management of malignant ureteral obstruction should be made on an individual patient basis.
\end{abstract}

Keywords: malignant obstruction, ureteral obstruction, cervical cancer, ureteral orifice, ureteral stent
Volume I Issue I - 2014

\author{
Joshua A Halpern, Peter N Schlegel, Bilal \\ Chughtai \\ Department of Urology, Weill Cornell Medical College of \\ Cornell University, USA
}

\begin{abstract}
Correspondence: Bilal Chughtai, Department of Urology, Weill Cornell Medical College of Cornell University, New York, USA,Email Bic9008@med.cornell.edu
\end{abstract}

Received: September II, 2014 | Published: October 18, 2014
Abbreviations: PCN, percutaneous nephrostomy tubes; MS, metallic ureteral stents

\section{Introduction}

A variety of pelvic, retroperitoneal or metastatic malignancies may cause ureteral obstruction either by direct invasion or external compression of the ureter. Ureteral obstruction is a cause of significant morbidity and mortality in cancer patients - these patients may suffer from acute or chronic pain, acute renal failure or subacute impairment of renal function, and they are at risk for infection due to an immunocompromised state. Obstructive uropathy has been associated with poor prognosis for the cancer patient.

Ureteral obstruction secondary to malignancy presents a distinct and challenging management scenario for the urologist. Success of traditional treatment modalities such as ureteral stents and percutaneous nephrostomy tubes ( $\mathrm{PCN})$ may be impaired by the presence of tumor or other disease-related factors. Furthermore, management must take into account unique factors in these patients, including life expectancy, quality of life implications for specific interventions, and the benefits of maintenance of renal function to all future treatment options for the patient's primary disease. Traditional therapies such as polymeric ureteral stents may be limited by disease progression and recurrent ureteral obstruction, and PCN may not be an optimal choice because of the need for an external appliance. Alternative therapies such as metallic ureteral stents (MS), parallel stent placement, and nephrovesical bypass have been proposed for this patient population with varying degrees of success.

We report on a 43 year old woman with invasive cervical cancer who presented with an incidental finding of hydroureter and was subsequently found to have malignant obstruction of her ureteral orifice on cystoscopic evaluation. A review of the literature regarding management of malignant ureteral obstruction follows.

\section{Case report}

A 43-year-old woman with a recent diagnosis of stage IIB cervical cancer was referred to our institution with vaginal bleeding and syncope. She had no flank pain, haematuria, dysuria, or lower urinary tract symptoms. Past medical history was unremarkable, and she had no family history of genitourinary pathology. On physical examination she had no flank tenderness and her urinalysis was normal. Her serum creatinine was $0.87 \mathrm{mg} / \mathrm{dL}$. She was admitted to the gynecologic oncology service for management of her cervical cancer and anemia.

MRI revealed a large pelvic mass extending from the cervix that was associated with severe right hydroureter, anterior displacement of the bladder, and bladder invasion at the level of the trigone (Figure 1). On cystoscopy, the bladder base, lateral walls, anterior wall and dome were free of lesions; however gross intraluminal tumor was protruding from the right ureteral orifice (Figure 2). A biopsy of the tumor was taken and the patient underwent right PCN placement. Pathologic analysis from the biopsy revealed adenocarcinoma consistent with an endocervical origin. Based on the pathologic findings, the patient's disease was classified as stage IVA and she was started on platinumbased chemotherapy and pelvic radiation.

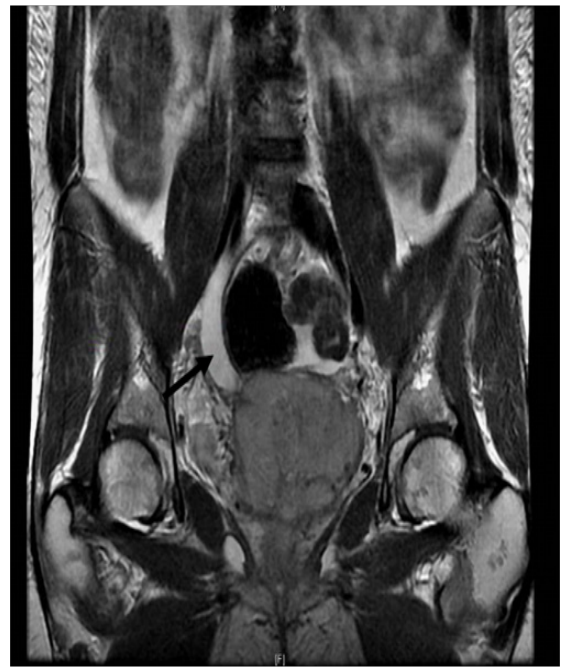

Figure I Magnetic resonance imaging (MRI) with contrast revealing severe right hydroureter (arrow).

Anterograde nephrostogram one month after placement of the PCN revealed persistent distal ureteral obstruction, and the PCN was replaced. Three months later, the patient developed apparent fever during chemotherapy with urine culture positive for Pseudomonas aeruginosa, and the patient underwent uncomplicated PCN exchange two days later with anterograde nephrostogram again noting persistent ureteral obstruction. No further urinary complications were noted. 


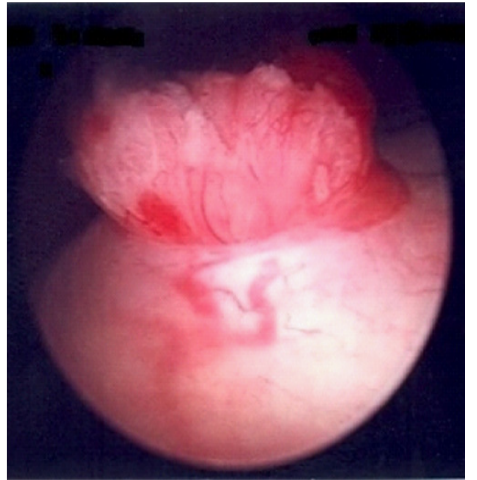

Figure 2 Right ureteral orifice with protrusion of papillary tumor of endocervical origin. Surrounding bladder mucosa is unremarkable.

\section{Discussion}

Malignant ureteral obstruction may be due to invasion of the ureter by cancer or external compression from a pelvic or retroperitoneal mass. Initial short-term management of obstructive uropathy due to malignancy may involve either retrograde ureteral stent placement or percutaneous nephrostomy with or without antegrade ureteral stent placement. Recently, authors have reported on a variety of novel approaches to malignant obstruction including deployment of metallic ureteral stents, parallel ureteral stents, and nephrocutaneous bypass.

In deciding upon interventions for patients with malignant ureteral obstruction, quality of life considerations are important. These patients often have short life expectancies, and the avoidance of pain, inconvenience, and psychosocial impairment may supersede the medical benefit of intervention. Monsky et al., ${ }^{1}$ retrospectively compared quality of life among patients with malignant obstruction managed with either stent or PCN. Those who underwent stent placement reported significantly higher incidence of both urinary symptoms and pain at 30 and 90 days post-procedure as compared to $\mathrm{PCN}$. However, PCN placement was associated with a higher incidence of minor complications and required more frequent exchange. Despite these patterns, there was no difference in responses to overall quality of life surveys between the two groups. Joshi et al., ${ }^{2}$ found that both interventions were equally associated with frequent complaints of pain, anxiety, and even depression. Therefore, asymptomatic patients with obstruction may be appropriate candidates for conservative management with serial laboratory and ultrasound evaluation.

Retrograde ureteral stenting has been the mainstay of palliative management with the majority of urologists favoring stent placement over percutaneous diversion as the primary treatment option. ${ }^{3}$ In a series of 157 patients who underwent attempted stent placement for initial management of malignant ureteral obstruction, Ganatra et al. ${ }^{4}$ reported an $84.7 \%$ initial success rate for retrograde placement of a ureteral stent. ${ }^{4}$ When accounting both for patients who failed initial stent placement and those who ultimately progressed to PCN due to stent obstruction, the overall retrograde stent failure rate was $35.7 \%$.

A number of risk factors have been associated with stent failure and poor survival in malignant obstruction. Pre-treatment renal insufficiency, the presence of metastatic disease, and invasion of malignancy into the bladder on cystoscopy, as in the above case presentation, are associated with a high rate of stent failure. ${ }^{5}$ Interestingly, patients with malignant obstruction secondary to a gynecologic malignancy have a more favorable prognosis than those with other cancers. The location of ureteral obstruction is also an important predictor of stent failure-free survival, as stents placed for middle or distal ureteral obstructions are more likely to fail. ${ }^{6}$

PCN is the primary alternative to ureteral stenting for initial management of malignant obstruction or in patients who progress to stent failure. Ishioka et al., ${ }^{7}$ reported the largest series of 140 patients undergoing PCN in the setting of advanced incurable malignancy. The most frequent complication related to $\mathrm{PCN}$ was dislodgement of the catheter requiring replacement in $19 \%$ of all patients.

In addition to polymeric stent and PCN placement, MS are of larger diameter, have theoretically increased tensile strength for resistance of external compression, can be left indwelling for longer periods than their polymeric predecessors, and have a small intravesical component, thereby sparing the patient from irritative bladder symptoms. Liatsikos et al., ${ }^{8}$ placed self-expandable MS via anterograde approach in 90 patients with ureteral obstruction secondary to malignancy. A large number of patients experienced complications including hyperplastic reaction within the stent, tumor in growth, stent encrustation, and stent migration. Overall, MS were primarily patent in just $51.2 \%$ of cases. Other iterations of the intra-ureteral metallic stent have been utilized in clinical practice, although none showing superior outcomes to the initial MS. Parallel stenting has also been proposed as an effective intervention for patients with ureteral stent failure. ${ }^{9}$ Patients who underwent parallel stenting had a lower stent failure rate at 3 months and lower post-procedural creatinine than those with stent exchange. This procedure is infrequently employed by urologists as it can be technically challenging.

Nephrovesical and nephrocutaneous bypass are intended to minimize the discomfort and burden of PCN and its accompanying external drainage bag requirements. Gerullis et al., ${ }^{10}$ performed nephrocutaneous bypass with a ventrally externalized subcutaneous tunneled pigtail catheter on 15 patients. There were no major perioperative complications, and all patients reported quality of life improvement following the operation. While these surgical bypass procedures are more invasive than traditional approaches, they may be appropriate in patients who cannot tolerate alternate therapies.

\section{Conclusion}

There are numerous interventions available for the management of ureteral obstruction secondary to advanced malignancy. Retrograde ureteral stent and PCN placement remain the two most frequently utilized approaches, although the development of metal ureteral stents and other stent combinations have broadened the treatment options and provide encouraging results. Without randomized clinical data to directly compare the safety and effectiveness of each of these interventions, decisions regarding management of malignant obstruction must be made on an individual basis with the assistance of risk stratification tools. Most importantly, patients should be educated regarding the quality of life implications of various therapies, and they should be integrally involved in the decision-making process.

\section{Acknowledgements}

None.

\section{Conflict of interest}

The author declares no conflict of interest. 


\section{References}

1. Monsky WL, Molloy C, Jin B, et al. Quality-of-life assessment after palliative interventions to manage malignant ureteral obstruction. Cardiovasc Intervent Radiol. 2013;36(5):1355-1363.

2. Joshi HB, Adams S, Obadeyi OO, et al. Nephrostomy tube or 'JJ' ureteric stent in ureteric obstruction: assessment of patient perspectives using quality-of-life survey and utility analysis. Eur Urol. 2001;39(6):695701.

3. Hyams ES, Shah O. Malignant extrinsic ureteral obstruction: a survey of urologists and medical oncologists regarding treatment patterns and preferences. Urology. 2008;72(1):51-56.

4. Ganatra AM, Loughlin KR. The management of malignant ureteral obstruction treated with ureteral stents. J Urol. 2005;174(6):2125-2128.

5. Chung SY, Stein RJ, Landsittel D, et al. 15-year experience with the management of extrinsic ureteral obstruction with indwelling ureteral stents. J Urol. 2004;172(2):592-595.
6. Yu SH, Ryu JG, Jeong SH, et al. Predicting factors for stent failure-free survival in patients with a malignant ureteral obstruction managed with ureteral stents. Korean J Urol. 2013;54(5):316-321.

7. Ishioka J, Kageyama Y, Inoue M, et al. Prognostic model for predicting survival after palliative urinary diversion for ureteral obstruction: analysis of 140 cases. J Urol. 2008;180(2):618-621.

8. Liatsikos EN, Karnabatidis D, Katsanos K, et al. Ureteral metal stents:10-year experience with malignant ureteral obstruction treatment. J Urol. 2009;182(6):2613-2617.

9. Chen HC, Shen SH, Wang JH, et al. Parallel second stent placement for refractory ureteral stent malfunction in malignant ureteral obstruction. $J$ Vasc Interv Radiol. 2011;22(7):1012-1016.

10. Gerullis H, Ecke TH, Schwartmann K, et al. Nephrocutaneous bypass in ureteral obstruction. Urology. 2010;76(2):480-485. 